PR-27

\title{
SYNTHESIS, CHARACTERIZATION AND CO2 SORPTION CAPACITY MEASUREMENTS OF SELECTED IONIC LIQUID POLYMERS
}

\author{
A. Vijaya Bhaskar Reddy, ${ }^{1}$ Muhammad Moniruzzaman, ${ }^{1,2}$ Bidyut Baran Saha, ${ }^{3}$ \\ N. Bakthavatchala Reddy, ${ }^{4}$ Grigory V Zyryanov ${ }^{4,5}$ \\ ${ }^{1}$ Centre of Research in Ionic Liquids (CORIL), Universiti Teknologi PETRONAS, \\ 32610 Seri Iskandar, Perak, Malaysia \\ ${ }^{2}$ Department of Chemical Engineering, Universiti Teknologi PETRONAS, \\ 32610 Seri Iskandar, Perak, Malaysia \\ ${ }^{3}$ International Institute for Carbon-Neutral Energy Research \\ (WPI-I2CNER), and Department of \\ Mechanical Engineering, Graduate School of Engineering, Kyushu University, \\ 744 Motooka, Nishi-ku, Fukuoka 819-0395, Japan \\ ${ }^{4}$ Ural Federal University, Chemical Engineering Institute, Yekaterinburg, \\ 620002, Russian Federation.
}

\section{${ }^{5}$ I. Ya. Postovskiy Institute of Organic Synthesis, Ural Division of the Russian Academy of Sciences, 22 \\ S. Kovalevskoy St., 620219 Yekaterinburg, Russian Federation. \\ *Corresponding authors, E-mail: m.moniruzzaman@utp.edu.my,vijay.dr555@gmail.com}

\begin{abstract}
Ionic liquids (ILs) technology has been emerged as the most prominent approach for the encapsulation of $\mathrm{CO}_{2}$ from flue gases, which is an important phenomena in oil \& natural gas industry during the gas sweetening process. The remarkable performance of IL polymers for the $\mathrm{CO}_{2}$ capture provided new opportunities to upgrade this technology in recent years. Therefore, in the present study, we have prepared different IL polymers namely poly(1-vinylimidazole), poly(1vinyl-3-ethylimidazolium bromide), poly(1-vinyl-3-ethylimidazolium bis (trifluoro methylsulfonyl))imide and zeolite incorporated poly(1-vinyl-3-ethylimidazolium bis (trifluoro methylsulfonyl)imide materials and evaluated their relative efficiency for the separation of $\mathrm{CO}_{2}$. The synthesized IL polymers were characterized by FT-IR, FE-SEM and EDX. The $\mathrm{CO}_{2}$ sorption experiments were conducted to evaluate the efficiency of synthesized IL polymers using gas sorption cell. The results revealed that, the IL-zeolite polymer has adsorbed highest amount of $\mathrm{CO}_{2}$ and recorded the highest pressure drop compared to other polymer ionic liquid and monomer ionic liquid materials.
\end{abstract}

